\title{
Switching Back to Normal Diet Following High-Fat Diet Feeding Reduces Cardiac Vulnerability to Ischaemia and Reperfusion Injury
}

\author{
Ben Littlejohns ${ }^{a}$ Hua Lin ${ }^{a} \quad$ Gianni D. Angelinia Andrew P. Halestrap ${ }^{b}$ \\ M. Saadeh Suleiman ${ }^{a}$
}

aBristol Heart Institute, School of Clinical Sciences, Faculty of Medicine and Dentistry, University of Bristol, 'bShool of Biochemistry, Faculty of Medical and Veterinary Sciences, University of Bristol, Bristol, UK

\section{Key Words}

Ischaemia/reperfusion • High-fat diet $•$ Mice $\cdot$ Heart $•$ Catalase $•$ Mitochondrial morphology • Cardiomyocyte $\bullet$ Calcium transient $\bullet$ Twitch contraction

\begin{abstract}
Background: We have recently shown that hearts of mice fed high-fat diet exhibit increased vulnerability to ischaemia and reperfusion (I/R) in parallel to changes in catalase protein expression, mitochondrial morphology and intracellular diastolic $\mathrm{Ca}^{2+}$. Aims: To determine whether switching from high-fat back to normal diet alters vulnerability to I/R and to investigate cardiac cellular remodelling in relation to the mechanism(s) underlying I/R injury. Methods and Results: Male C57BL/6J mice were fed a high-fat diet for 19-22 weeks; after which a subset of mice was switched back to normal diet for 4-6 weeks. Hearts from mice switched back to normal diet were more resistant to reperfusion injury compared to hearts from mice fed only high-fat diet. This was associated with a significant reversal in catalase expression (western blotting) and recovery of size and density of mitochondria (electron microscopy). In contrast, switching back to normal diet did not alter cardiomyocyte contractility or $\mathrm{Ca}^{2+}$ transients compared to high-fat diet. Conclusion: This study shows for the first time that switching the diet from high-fat back to normal reduces vulnerability to I/R. This effect is associated with changes in catalase levels and mitochondrial morphology without altering cardiomyocyte contractility or $\mathrm{Ca}^{2+}$ transients.
\end{abstract}




\section{Introduction}

We have recently shown that hearts from mice fed high-fat diet, in the absence of obesity associated co-morbidities, exhibit increased vulnerability to ischaemia and reperfusion (I/R) [1]. This was attributed to altered oxidative state, mitochondrial modifications and changes in $\mathrm{Ca}^{2+}$ handling. Interestingly, obesity-generating high-fat diet has also been shown to increase vulnerability of hearts to I/R [2-4]. Whether the high-fat diet increased vulnerability to I/R can be reversed by returning back to a normal diet for a relatively short period is not presently known. However, there is evidence showing that switching diet back to normal after periods of high-fat diet feeding is associated with changes back to normal conditions [5]. Such changes depend on the duration and magnitude of weight gain - factors that are often confounded because different degrees of obesity are created by varying the length of exposure to the high energy diet. For example, generating varying degrees of weight gain before switching back to normal diet can normalise metabolic abnormalities [6]. Furthermore, obesity resulting from short periods of high-fat feeding could not be fully reversed in terms of body weight and fat even after weeks of switching back to normal diet. In contrast, more recent evidence has shown that returning mice to normal diet after a long period of obesogenic high-fat diet resulted in a return of the weight to the normal level [7]. Nonetheless, several studies have demonstrated the reversibility of body weight gain when mice fed high-fat diet were switched back to normal diet $[8,9]$. Other key changes in the left ventricle were also reversed upon returning to normal diet including markers of inflammation, oxidised proteins, markers of apoptosis, anti-oxidants and antifibrotic markers [7]. High-fat diet induced changes in blood chemistry (e.g. hyperglycaemia, hyperinsulinaemia, hypertriglyceridaemia and hypercholesterolaemia) are also fully reversible by returning to normal diet [9].

These studies demonstrate that many of the changes associated with obesity and high-fat diet are reversible. However, to the best of our knowledge, there are no reports showing that switching from high-fat (with or without obesity) to normal diet can reverse the increased vulnerability to I/R. Therefore, the aim of this work was to determine whether, in our recently characterised mouse model of non-obesogenic high-fat diet [1], the vulnerability to I/R can be changed by switching back to normal diet. We have also monitored myocardial cellular remodelling with emphasis on changes in catalase expression, mitochondrial morphology and $\mathrm{Ca}^{2+}$ handling. The reason for focusing on these endpoints was because they were the ones that correlated with increased vulnerability to I/R in the high-fat diet group [1].

\section{Materials and Methods}

More details of materials and methods can be found in our earlier publication [1].

\section{Animals and Diet}

Breeding, maintenance and feeding of C57BL/6J male mice as well as weight monitoring and clinical chemistry were all carried out at Charles River facilities (Charles River, Margate, UK). A summary of the feeding protocols is shown in Scheme 1. During the feeding protocols the mice were given ad libitum access to food and water and maintained on a $12 \mathrm{~h}$ light/dark cycle. Mice were fed standard murine chow diet post weaning until 6 weeks of age and they were either maintained on the standard chow (normal diet mice) or they were switched to high-fat diet (high-fat diet mice) for a further 20-22 weeks. The normal diet contained $13 \%$ calories from fat, $22 \%$ calories from protein and $65 \%$ calories from carbohydrate. The high-fat diet contained $0.17 \%$ calories from cholesterol and low sucrose content and consisted of (\% calories) $45 \%$ fat, $18 \%$ protein and $37 \%$ carbohydrate (Special Diets Services, UK, code: 821424). The gross energy content for high-fat and normal diets was 19.67 and $16.54 \mathrm{~kJ} \cdot \mathrm{g}^{-1}$, respectively. The detailed components of the diet can be found in [1]. A group of these mice were switched back to normal diet for 4-6 weeks after 19-20 weeks of high-fat diet (high-fat/normal diet mice). The experiments in this additional group of mice were carried out simultaneously with the two previous groups of mice which we have already published the data 


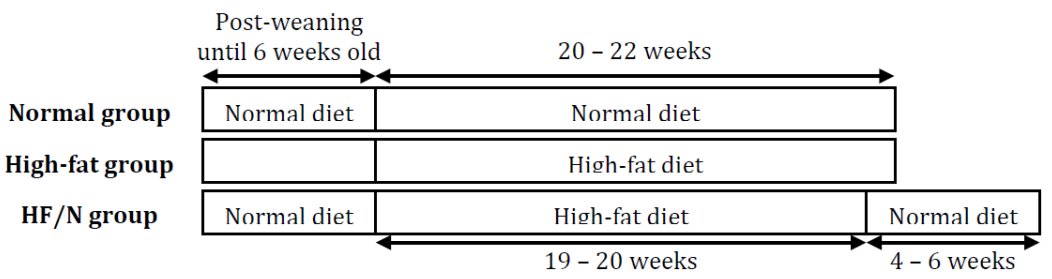

Scheme 1. The feeding protocols. HF/N = high-fat followed by normal diet

for [1]. The feeding duration in the high-fat/normal group was slightly different to avoid using additional groups of mice. Essentially it was a balance between keeping the feeding duration similar and having a similar overall age of the mice. As the mice were all adults, the impact of age differences is minimal.

\section{Clinical chemistry measurements}

Tail vein blood was taken from non-fasted mice and pooled (one pooled sample was from three animals) to measure blood chemistry components performed by Charles River (Margate, UK).

\section{$I / R$ injury in isolated perfused hearts}

Excised hearts were cannulated in a Langendorff mode and perfused at a constant filling pressure of $60 \mathrm{mmHg}$ with Krebs-Henseleit buffer and gassed with $95 \% \mathrm{O}_{2}$ and $5 \% \mathrm{CO}_{2}\left(\mathrm{pH} \mathrm{7.4}, 37^{\circ} \mathrm{C}\right)$. The Krebs-Henseleit buffer contained, in mM, $120 \mathrm{NaCl}, 25 \mathrm{NaHCO}_{3}, 11$ D-glucose anhydrous, $1.2 \mathrm{KH}_{2} \mathrm{PO}_{4}, 1.2$ $\mathrm{MgSO}_{4} \cdot 7 \mathrm{H}_{2} \mathrm{O}, 4.8 \mathrm{KCl}$ and $2 \mathrm{CaCl}_{2}$. After a 30 min stabilisation period the hearts were rendered ischaemic for $40 \mathrm{~min}$ (no flow global ischaemia) followed by $120 \mathrm{~min}$ reperfusion. Triphenyl tetrazolium chloride (TTC) staining was used to determine the infarct size as described previously [10]. Vascular dysfunction was monitored by comparing the extent of recovery in coronary flow rate. Coronary effluent was collected at different time points and used to measure creatine kinase using a commercially available kit from Randox, UK (CK113). Total creatine kinase release was determined by calculating the area under the curve.

\section{Western blotting}

Primary antibodies used were catalase (1:2000, Abcam) and the loading control glyceraldehyde3-phosphate dehydrogenase (GAPDH) (1:10,000, Cell Signaling). Blots were then incubated with an appropriate horseradish peroxidase conjugated secondary antibody (1:10,000, GE Healthcare Life Sciences) and proteins were visualised using the enhanced chemiluminescence system. Protein bands were quantified by densitometry with Image $1.46 \mathrm{r}$ software.

\section{Measurement of cardiac mitochondrial morphology}

Mitochondrial morphology was assessed as described previously [1]. In brief, fixed cardiac tissue was processed for electron microscopy and cut into $70 \mathrm{~nm}$ sections with the cardiomyocytes in longitudinal plane. The mitochondria were viewed with a Tecnai 12 bioTWIN transmission electron microscope (FEI, Netherlands) and analysed using ImagePro Plus software (Media Cybernetics, USA).

\section{Work on isolated cardiomyocytes}

Ventricular cardiomyocytes were isolated and superfused at a rate of $1.3 \mathrm{~mL} \cdot \mathrm{min}^{-1}$ with HEPES buffer solution $\left(32-33^{\circ} \mathrm{C}\right)$, consisting of in $\mathrm{mM}, 137 \mathrm{NaCl}, 5 \mathrm{KCl}, 1.2 \mathrm{MgSO}_{4} \cdot 7 \mathrm{H}_{2} \mathrm{O}, 1.2 \mathrm{NaH}_{2} \mathrm{PO}_{4} \cdot 2 \mathrm{H}_{2} \mathrm{O}, 20 \mathrm{HEPES}$, 15 D-glucose anhydrous and $2 \mathrm{CaCl}_{2}(\mathrm{pH}$ 7.4) $[1,11]$. Superfused cardiomyocytes were field stimulated and contractility measured using an edge tracking device. The ratiometric dye Fura-2 was used to monitor intracellular $\mathrm{Ca}^{2+}$ concentrations $\left(\left[\mathrm{Ca}^{2+}\right]_{\mathrm{i}}\right)$ and was measured using photometry (Photon Technology International, USA).

\section{Data analysis}

Data were analysed using Prism 5 Version 5.01 software (GraphPad, USA) and presented as mean \pm SEM where appropriate. Data were tested for normal distribution using Kolmogorov-Smirnov test and equal variance using F-test. One-way ANOVA with Bonferroni's post-hoc test was performed or if the data did not have equal variance and normal distribution then Kruskal-Wallis test with Dunn's post-hoc test 
Table 1. Characteristics of the mice and blood chemistry analysis. Data are presented as mean \pm SEM. For blood for chemistry analysis each $\mathrm{n}$ was a pooled sample from three mice. Data were analysed using the appropriate statistical test. $*=\mathrm{P}<0.05$ vs. normal diet and $\#=\mathrm{P}<0.05$ vs. high-fat diet

\begin{tabular}{lccc}
\hline Measurement & Normal diet & High-fat diet & High-fat/normal diet \\
\hline Body weight $(\mathrm{g})$ & $31.2 \pm 0.2$ & $32.2 \pm 0.3^{*}$ & $31.5 \pm 0.4$ \\
& $(\mathrm{n}=158)$ & $(\mathrm{n}=153)$ & $(\mathrm{n}=32)$ \\
& $0.53 \pm 0.02$ & $1.19 \pm 0.11^{*}$ & $0.79 \pm 0.08^{*}$ \\
Epididymal fat pad weight $(\mathrm{g})$ & $(\mathrm{n}=55)$ & $(\mathrm{n}=34)$ & $(\mathrm{n}=11)$ \\
& $219 \pm 5$ & $215 \pm 6$ & $216 \pm 7$ \\
Wet heart weight $(\mathrm{mg})$ & $(\mathrm{n}=55)$ & $(\mathrm{n}=34)$ & $(\mathrm{n}=11)$ \\
& $3.25 \pm 0.20$ & $5.06 \pm 0.52^{*}$ & $3.43 \pm 0.29^{*}$ \\
Cholesterol $(\mathrm{mM})$ & $(\mathrm{n}=4)$ & $(\mathrm{n}=4)$ & $(\mathrm{n}=4)$ \\
& $120 \pm 15$ & $205 \pm 26^{*}$ & $156 \pm 16$ \\
Triglycerides $\left(\mathrm{mg} \cdot \mathrm{dL}^{-1}\right)$ & $(\mathrm{n}=4)$ & $(\mathrm{n}=4)$ & $(\mathrm{n}=4)$ \\
& $9.26 \pm 0.64$ & $7.54 \pm 0.72$ & $8.90 \pm 0.40$ \\
Non-fasting glucose $(\mathrm{mM})$ & $(\mathrm{n}=4)$ & $(\mathrm{n}=4)$ & $(\mathrm{n}=4)$ \\
& & &
\end{tabular}
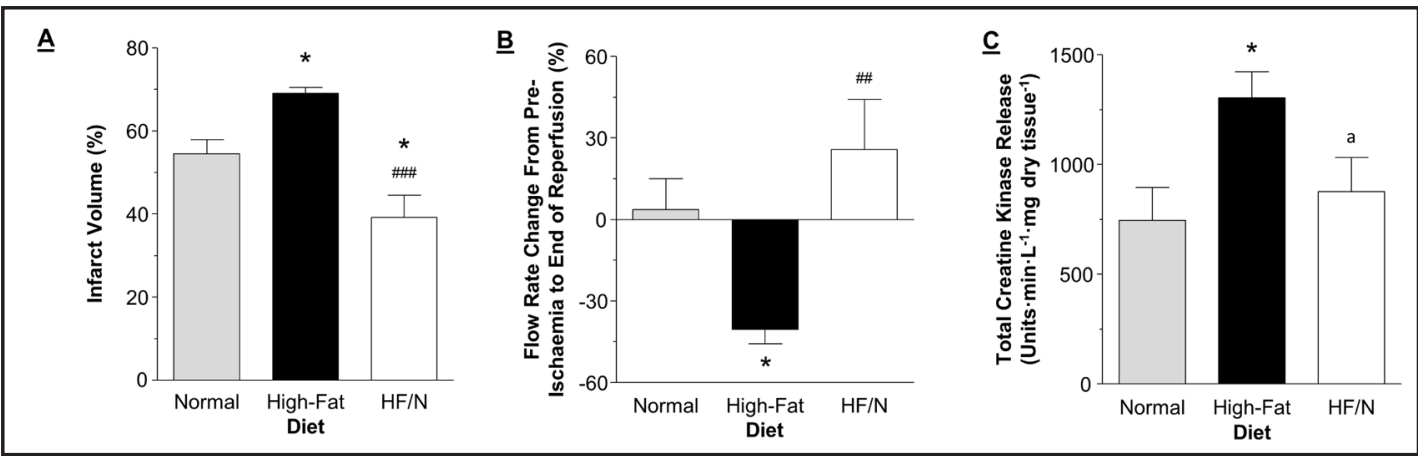

Fig. 1. Markers of injury during I/R. A) Infarct volume quantified from the heart slices. B) Flow rate change from pre-ischaemia to the end of reperfusion. C) Total creatine kinase release collected in the coronary effluent during reperfusion. Data are presented as mean \pm SEM $(n=5-7$ hearts). HF/N = high-fat/normal diet. Data were analysed using one-way ANOVA with Bonferroni's post-hoc test. ${ }^{*}=\mathrm{P}<0.05$ vs. normal diet and $\# \# \#=\mathrm{P}<0.001$ and \#\# = $\mathrm{P}<0.01$ vs. high-fat diet. $\mathrm{a}$ is $\mathrm{P}=0.05 \mathrm{vs}$. high-fat diet. The infarct data for normal and high-fat diets were taken from [1].

was performed. Two-way ANOVA with Bonferroni's post-hoc test was used on data with two independent variables. All statistical tests were performed as unpaired, two-tailed and a P-value less than 0.05 was assumed to be significantly different.

\section{Results}

This study represents an extension of our recently published work investigating the effect of high-fat diet (independent of obesity associated co-morbidities) on cardiac remodelling and vulnerability to I/R [1]. The data presented in this paper are largely focused on a group of mice fed high-fat diet (19-20 weeks) and then switched back to normal diet (4-6 weeks). For the sake of clarity, we used some of the published data from mice fed normal and high-fat diet. We have permission to use this data and this has been pointed out and acknowledged in the text.

\section{Characteristics and blood chemistry analysis of mice fed high-fat/normal diet}

Feeding C57BL/6J male mice a non-obesogenic high-fat diet for approximately 20 weeks slightly increased the body weight and this was reversed in the high-fat/normal diet mice (Table 1). Despite the body weight returning back to the normal diet range, the epididymal fat pads were relatively lower than the high-fat diet group but still significantly heavier than the normal diet (Table 1). There was a significant reduction in blood cholesterol levels in the high-fat/normal diet compared to the high-fat diet and blood triglyceride levels in the highfat/normal diet group were no longer different to the normal diet group (Table 1). The wet heart weight and non-fasting blood glucose were similar between the normal, high-fat and high-fat/normal diets (Table 1). The normal and high-fat diet data was taken from [1]. 
Fig. 2. Cardiac catalase protein expression. Cardiac catalase protein expression determined using western blot. Catalase was normalised to GAPDH. Data are presented as mean \pm SEM ( $n=3-6$ hearts). HF/N = high-fat/normal diet. Data were analysed using one-way ANOVA with Bonferroni's post-hoc test. ${ }^{* *}=\mathrm{P}<0.01$ vs. normal diet and ${ }^{\# \# \#}=\mathrm{P}<0.001$ vs. high-fat diet.

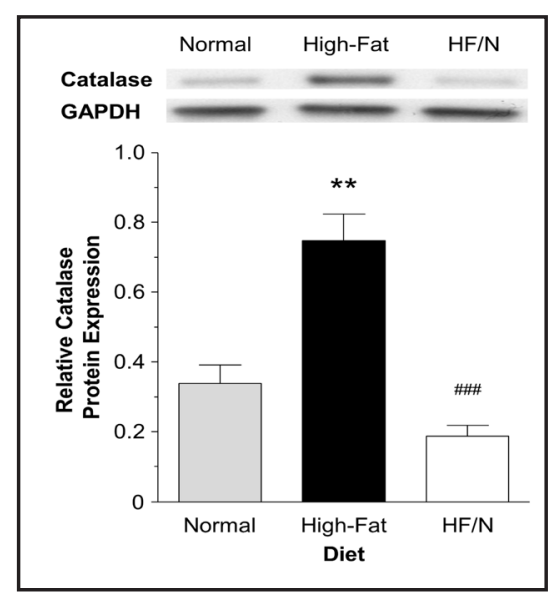

Fig. 3. Representative electron micrographs and characteristics of mitochondrial morphology. Representative electron micrographs from hearts of mice fed normal (A), high-fat diet (B) and high-fat/normal diet (C). Individual mitochondrion area (D) and length (E), mitochondrial coverage of myofilament area $(F)$ and number of lipid droplets per unit area (G) assessed using transmission electron micrographs. Data are presented as mean \pm SEM $(n=$ 4 hearts and $\geq 900$ mitochondria per heart from $\geq 10$ electron micrographs per heart). HF/N = high-fat/normal diet. Data were analysed using Kruskal-Wallis test with Dunn's post-hoc test (D-E) and one-way ANOVA with Bonferroni's post-hoc test (FG). ${ }^{* * *}=\mathrm{P}<0.001$ and $*=\mathrm{P}<$ 0.05 vs. normal diet and ${ }^{\# \#}=\mathrm{P}$ $<0.001$ and $^{\#}=\mathrm{P}<0.05$ vs. highfat diet. The data for mitochondrial area, length and coverage for normal and high-fat diets were taken from [1].

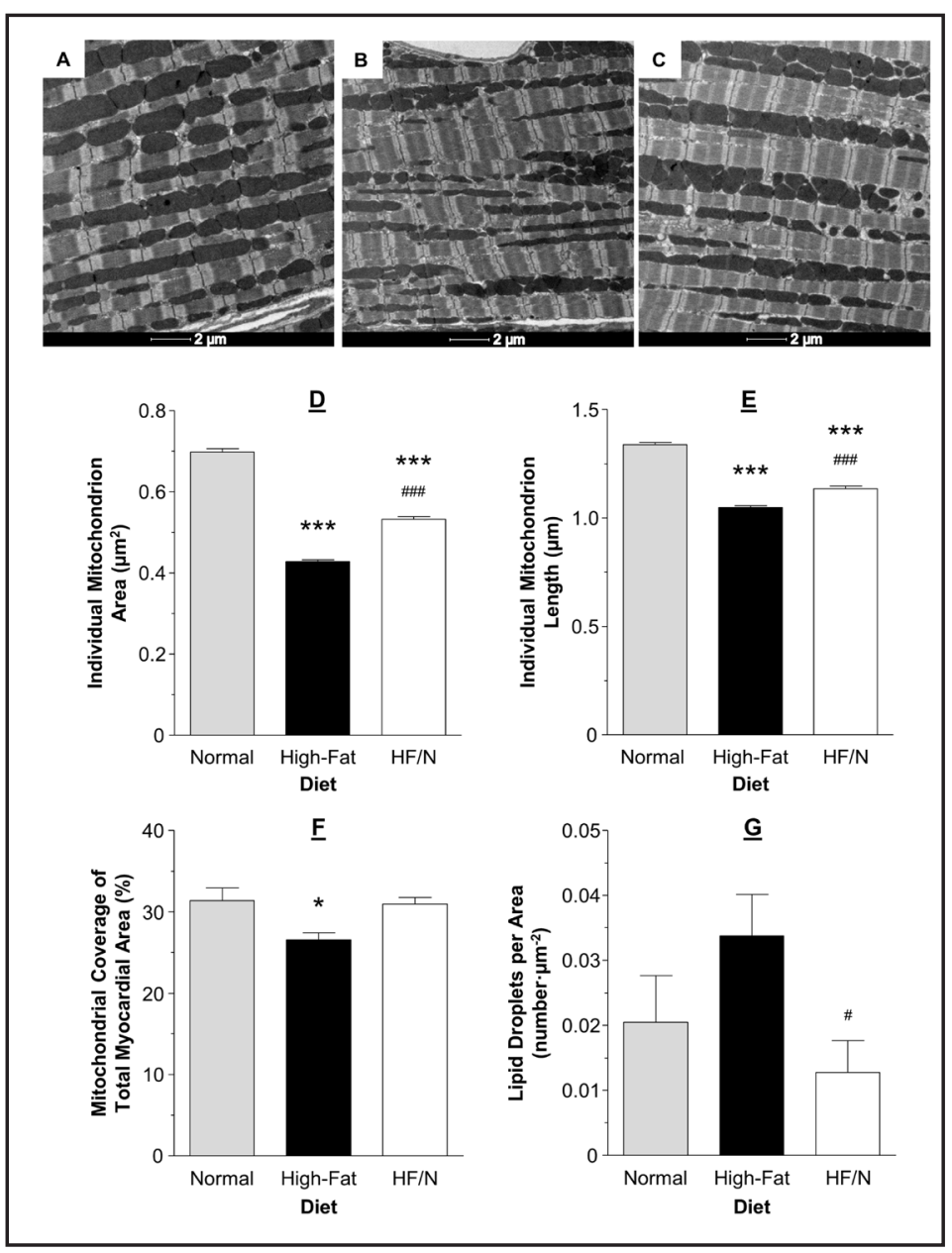

The effects of I/R on hearts isolated from mice fed high-fat/normal diet

Hearts isolated from mice fed high-fat/normal diet and subjected to I/R had significantly less infarct volume compared to the high-fat diet group, $\mathrm{P}<0.001$ and lower than normal, $\mathrm{P}$ $<0.05$ (Fig. $1 \mathrm{~A}$ ). This was associated with significantly better preservation of coronary flow rate, $\mathrm{P}<0.01$ (Fig. $1 \mathrm{~B}$ ) and a total release of creatine kinase which was similar to the value for normal diet (Fig. 1 C). 


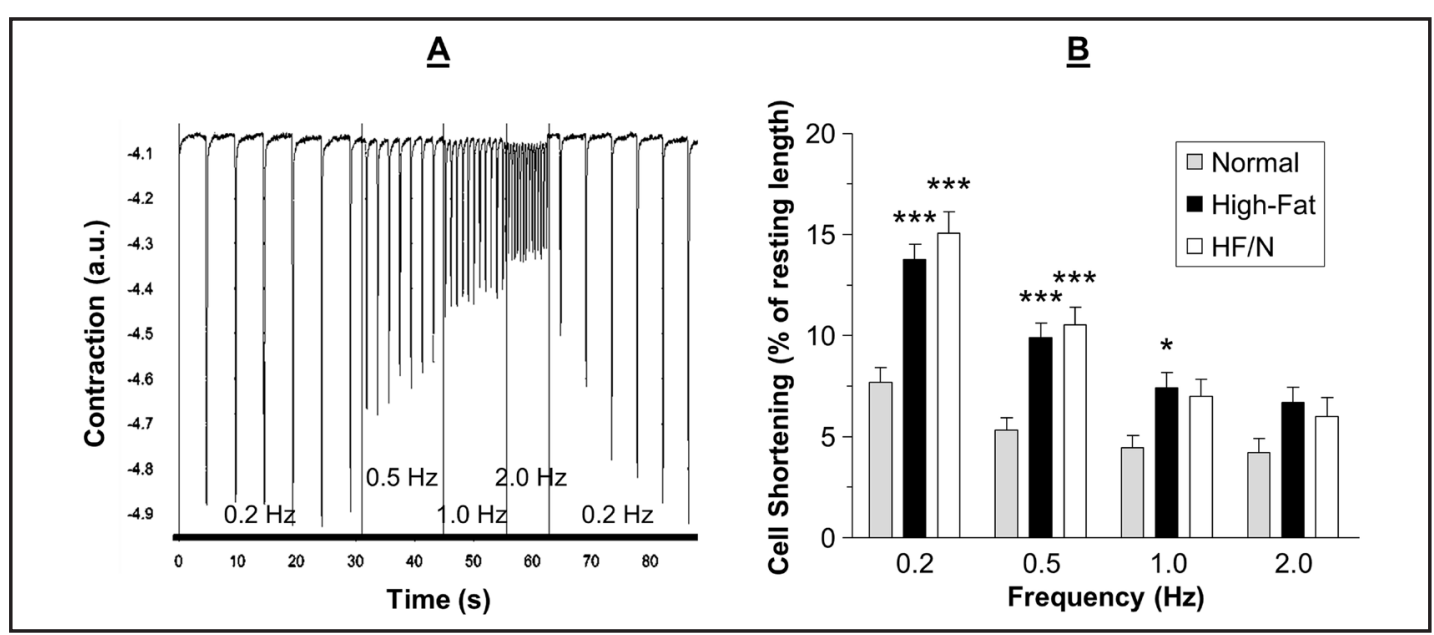

Fig. 4. Representative contractility trace and analysis. Data were recorded using an edge tracking device to measure contractility of cardiomyocytes field stimulated at $0.2,0.5,1.0$ and $2.0 \mathrm{~Hz}$. A) Representative contractility trace of cardiomyocytes isolated from mice fed a high-fat/normal diet with the frequencies of stimulation indicated. B) The shortening of cardiomyocytes at different frequencies. Data are presented as mean \pm SEM ( $\mathrm{n}=22-41$ cardiomyocytes from 5-6 hearts). HF $/ \mathrm{N}=$ high-fat $/$ normal diet and a.u. = arbitrary units. Data were analysed using two-way ANOVA with Bonferroni post-hoc test. ${ }^{* * *}=\mathrm{P}<0.001$ and ${ }^{*}=\mathrm{P}<$ 0.05 vs. normal diet.

Cardiac catalase protein expression in mice fed high-fat/normal diet

In the high-fat/normal diet group the cardiac catalase protein expression was significantly lower compared to the high-fat diet group and tended to be lower than in normal diet although this was not statistically significant (Fig. 2).

Cardiac mitochondrial morphology in mice fed high-fat/normal diet

The mitochondria in the high-fat/normal diet group were significantly larger and longer compared to the high-fat diet group, $\mathrm{P}<0.001$ (Fig. $3 \mathrm{~B}-\mathrm{E}$ ). This was a partial recovery as the mitochondria in the high-fat/normal diet group were still smaller and shorter compared to the normal diet, $\mathrm{P}<0.001$ (Fig. $3 \mathrm{~A}-\mathrm{E}$ ). The mitochondrial density (the total mitochondrial area as a percentage of total myofilament area) was increased in the high-fat/normal diet group compared to the high-fat diet group, but similar to the normal diet group (Fig. $3 \mathrm{~F}$ ). There was a drop in the number of lipid droplets seen in electron micrographs between the high-fat diet and high-fat/normal diet groups, $\mathrm{P}<0.05$ (Fig. $3 \mathrm{G}$ ).

Contractility and $\mathrm{Ca}^{2+}$ transients in cardiomyocytes isolated from mice fed high-fat/ normal diet

A representative trace of cardiomyocyte contractility is shown in Fig. 4A. The twitch contractions of the cardiomyocytes were significantly larger in the high-fat diet group compared to the normal diet group, except at $2.0 \mathrm{~Hz}$, and this difference was maintained in the high-fat/normal diet group at 0.2 and $0.5 \mathrm{~Hz}, \mathrm{P}<0.05$ (Fig. 4 B). The time to the peak contraction and time to $90 \%$ recovery of the resting cell length following a contraction were not different between the normal, high-fat diet and high-fat/normal diet groups, except that at $0.2 \mathrm{~Hz}$ the time to the contraction peak was slower in the high-fat/normal diet compared to the normal diet and high-fat diet groups (data not shown).

For a representative trace of $\mathrm{Ca}^{2+}$ transient measurements see Fig. 5A. The area under the $\mathrm{Ca}^{2+}$ transient was significantly larger in the high-fat diet group compared to the normal diet group, except at $2.0 \mathrm{~Hz}$, and this difference was maintained in the high-fat/normal diet group at 0.2 and $0.5 \mathrm{~Hz}, \mathrm{P}<0.05$ (Fig. $5 \mathrm{~B}$ ). There was no difference in the amplitude of the $\mathrm{Ca}^{2+}$ transient between the normal and high-fat diets. However, the amplitude was lower in 


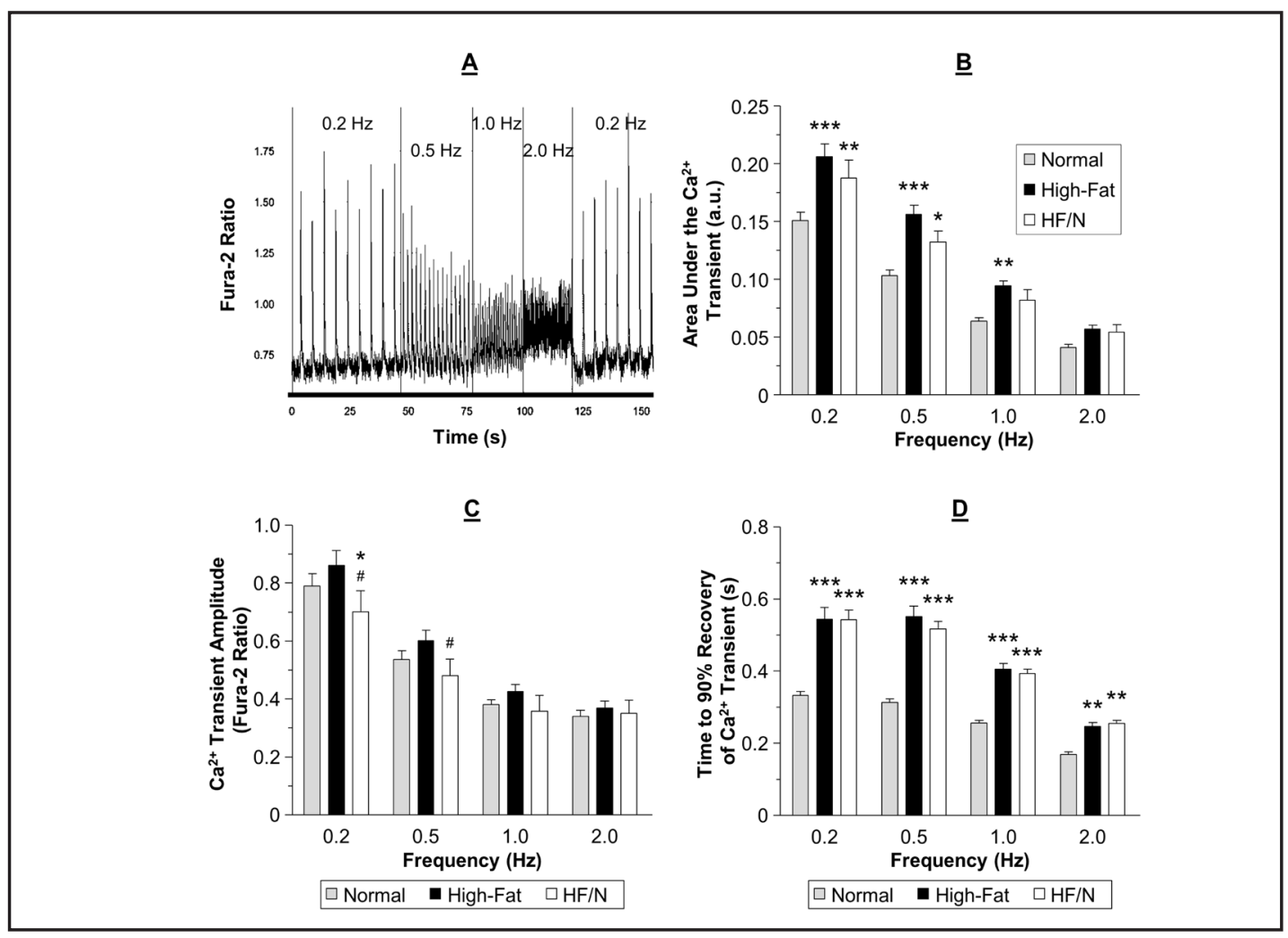

Fig. 5. Representative $\mathrm{Ca}^{2+}$ transient trace and analysis. Data were recorded using Fura-2 AM fluorescence to measure $\mathrm{Ca}^{2+}$ transients of cardiomyocytes field stimulated at $0.2,0.5,1.0$ and $2.0 \mathrm{~Hz}$. A) Representative $\mathrm{Ca}^{2+}$ transient trace of cardiomyocytes isolated from mice fed a high-fat/normal diet with the frequencies of stimulation indicated. B) The area under the $\mathrm{Ca}^{2+}$ transient. C) The amplitude of the $\mathrm{Ca}^{2+}$ transient. D) Time for the $\mathrm{Ca}^{2+}$ transient to return to $90 \%$ of the diastolic level. Data are presented as mean $\pm \mathrm{SEM}(\mathrm{n}=$ 22-32 cardiomyocytes from 3-4 hearts). HF/N = high-fat/normal diet and a.u. = arbitrary units. Data were analysed using two-way ANOVA with Bonferroni post-hoc test. ${ }^{* *}=\mathrm{P}<0.001,{ }^{* *}=\mathrm{P}<0.01$ and $*=\mathrm{P}<0.05$ vs. normal diet and ${ }^{\#}=\mathrm{P}<0.05$ vs. high-fat diet.

the high-fat/normal diet group at $0.2 \mathrm{~Hz}$ compared to the both other groups and at $0.5 \mathrm{~Hz}$ compared to the high-fat diet group (Fig. 5 C). The normal and high-fat diets had a similar time to peak at all frequencies tested. The only frequency that the high-fat/normal diet had a slower time to peak of the $\mathrm{Ca}^{2+}$ transient was at $0.5 \mathrm{~Hz}$ (data not shown). The time to 90 $\%$ recovery of the $\mathrm{Ca}^{2+}$ transient was significantly longer in the high-fat diet compared to normal diet and this difference was maintained in the high-fat/normal diet group, $\mathrm{P}<0.05$ (Fig. 5 D). After feeding mice the high-fat/normal diet the diastolic $\left[\mathrm{Ca}^{2+}\right]_{\mathrm{i}}$ remained at the level of the high-fat diet group (data not shown) which is higher compared to normal diet (see [1]).

\section{Discussion}

We recently used a non-obesogenic (little weight gain and no insulin resistance) mouse model of high-fat feeding (raised blood cholesterol and triglycerides) to show that isolated hearts from these mice have increased vulnerability to I/R compared to mice fed a normal diet [1]. The proposed mechanism for increased I/R injury was a combination of elevated catalase level and inability to adapt to an increase in oxidative stress, fragmentation of mitochondria, reduced mitochondrial density and elevated diastolic $\left[\mathrm{Ca}^{2+}\right]_{\mathrm{i}}[1]$. In this study we show for the first time that switching the diet from high-fat back to normal for a few 
weeks significantly reduces the vulnerability of isolated hearts to I/R. This period of normal diet was associated with reduced blood lipids, reversal of catalase protein expression and alterations in mitochondrial morphology towards that of mitochondria in normal diet mice. However, the contractile characteristics and $\mathrm{Ca}^{2+}$ handling measurements in cardiomyocytes remained similar to the high-fat diet.

Switching from high-fat back to normal diet reduces vulnerability to I/R: the role of catalase and mitochondrial morphology

In this study, we found that switching the diet from high-fat to normal significantly reduces vulnerability to I/R. Reactive oxygen species (ROS) production and $\mathrm{Ca}^{2+}$ loading are key triggers of reperfusion injury [12]. Key in combating the increase in ROS levels are the anti-oxidant enzymes including catalase, superoxide dismutase, peroxiredoxins and glutathione peroxidase. In our earlier work, we found that high-fat diet increases the protein expression of cardiac catalase and that the level of this enzyme does not change during I/R. This was in contrast to normal diet, where this anti-oxidant enzyme markedly increased during I/R and improved the anti-oxidant capacity in the myocardium as demonstrated by relatively less increase in lipid peroxidation [1]. Therefore it was suggested that the inability of catalase to be further augmented during I/R in the high-fat diet group would reduce the capacity of the myocardium to scavenge ROS produced during I/R injury. In this study, we found that switching the diet from high-fat back to normal significantly reduced catalase protein expression back to the normal diet level (Fig. 2). This suggests that these hearts will have an improved anti-oxidant reserve and reduced susceptibility to I/R [1]. Although there are other reports showing that high-fat diet triggers an up regulation of catalase protein expression and activity [13] we are unaware of any reports showing that this can be reversed by switching back to normal diet. An interesting observation was the finding that cardiac catalase levels in the high-fat/normal diet group tended to reverse past the normal diet level. Moreover, the infarct size is actually smaller in the high-fat/normal diet compared to the normal and the recovery in flow rate also tended to be better the normal. Whether catalase is responsible for this effect is not presently known and this requires further investigation.

Altered mitochondrial morphology has been demonstrated to have an impact on vulnerability to I/R. Larger mitochondria are implicated in better protection against I/R possibly by being able to accommodate more $\mathrm{Ca}^{2+}$ before mitochondrial permeability transition pore opening which is a key determinant of I/R injury [12, 14]. In addition, mitochondrial fragmentation was shown to be responsible for I/R injury [15]. This is consistent with our earlier study showing that high-fat diet was associated with smaller and shorter mitochondria (fragmented mitochondria) and increased I/R injury [1]. Furthermore, we found high-fat diet to be associated with less mitochondrial coverage of myofilament area. Thus it was concluded that high-fat diet altering of mitochondrial morphology/ density predisposes the heart to reperfusion injury. In this study, we found that switching the diet back to normal, significantly reversed these changes (Fig. 3) and therefore might be responsible for improved cardioprotection. These changes were only a partial recovery as the mitochondria were still shorter and smaller compared to the normal diet; however, despite the mitochondria being smaller and shorter the density had returned to the normal diet level.

The changes in catalase and mitochondrial morphology are reversed in the high-fat/ normal diet group compared to high-fat diet and these are likely to be responsible for decreased I/R injury. However, the possibility that other cellular changes also contribute to this cannot be excluded.

Cardiomyocyte $\mathrm{Ca}^{2+}$ transients and contractility do not change in response to switching from high-fat back to normal diet

In our earlier work we found that high-fat diet was associated with a raised cytosolic $\mathrm{Ca}^{2+}$ content in cardiomyocytes compared to the normal diet group and this is known to be trigger for increased I/R injury [12]. Surprisingly, switching the diet from high-fat back to 
normal was not associated with changes in cytosolic $\mathrm{Ca}^{2+}$ or in any of the characteristics of the $\mathrm{Ca}^{2+}$ transients suggesting that our original proposal that the high-fat diet increased vulnerability to I/R could be due to $\mathrm{Ca}^{2+}$ regulation may not be correct.

In this study, we report the characteristics of cardiomyocyte twitch contractions from normal, high-fat and high-fat/normal diet groups. In parallel to our finding for $\mathrm{Ca}^{2+}$ transients, we found that cardiomyocyte contractility changes in high-fat diet and that the changes are not reversed, on the whole, upon switching back to normal diet (Fig. 4). The cardiomyocyte twitch contractions were larger in high-fat diet compared to normal diet and this was maintained in the high-fat/normal diet group. At the higher frequencies studied the difference between the groups became smaller; a likely explanation for this could be reduced sensitivity of the detection system resulting from having smaller amplitude at higher rates. Increased amplitude of cardiomyocyte contractility has already been reported in mice fed a high-fat high-sucrose diet which induced obesity and diabetes. These mice demonstrated an increased peak shortening in cardiomyocytes isolated from mice at 5 and 7 months of highfat feeding [16]. Heart failure induced in rats by coronary artery ligation and then fed a high saturated fat diet also had improved contractile function in vivo compared to the normal diet; however the saturated fat diet had no effect on contractility in the absence of heart failure [17]. The novel finding here is that by switching back to normal diet the increased amplitude of twitch contractions is not reversed.

Despite the larger twitch contractions there was no increase in the $\mathrm{Ca}^{2+}$ transient amplitude. However, there was a larger area under the $\mathrm{Ca}^{2+}$ transient which is likely to lead to the larger twitch contractions in the cardiomyocytes isolated from high-fat fed mice compared to normal diet fed mice. Other possible mechanisms for the increased twitch contractions are altered sensitivity of the myofilaments to $\mathrm{Ca}^{2+}$ and changes in the viscoelastic properties of the cardiomyocyte in response to high-fat diet. The greater area under the $\mathrm{Ca}^{2+}$ transient can be attributed to the slower reuptake of $\mathrm{Ca}^{2+}$ following a $\mathrm{Ca}^{2+}$ transient. As we have already reported, mice fed a high-fat diet have reduced cardiac phospholamban phosphorylation at site Ser16 [1]. In the high-fat/normal diet group the phospholamban phosphorylation was similar to the high-fat diet group (unpublished data). In rodents, the majority of the $\mathrm{Ca}^{2+}$ released from the sarcoplasmic reticulum during a $\mathrm{Ca}^{2+}$ transient is taken back up into the sarcoplasmic reticulum via SERCA [18]. The remaining $\mathrm{Ca}^{2+}$ is extruded from the cell by the $\mathrm{Na}^{+} / \mathrm{Ca}^{2+}$ exchanger, the $\mathrm{Ca}^{2+}$ ATPase or taken up into the mitochondria. The rate of the $\mathrm{Ca}^{2+}$ transient recovery therefore largely depends on SERCA and its activity. Phospholamban is a regulator of SERCA activity and therefore the slower $\mathrm{Ca}^{2+}$ transient in the high-fat diet group is likely to be due to the reduced phospholamban phosphorylation.

However, one thing to note is that the increase in amplitude of contraction seen in the high-fat diet group compared to normal diet group was not observed in echocardiographic measurements as there was no change in ejection fraction or fractional shortening (see [1]). The stimulation rate used in the isolated cardiomyocytes was far from the physiological rate seen in vivo; when the stimulation frequency reached $2.0 \mathrm{~Hz}$ there was no longer a significantly increased twitch contraction, therefore it is possible that the changes observed in the cardiomyocytes do not represent what occurs in vivo. It is not known what the physiological significance of the increased twitch contractions at low frequencies is. It is also not known if the $\mathrm{Ca}^{2+}$ and contractility changes could be reversed if the mice were maintained on the normal diet for a longer period following the high-fat diet. Nonetheless, as the vulnerability to I/R was reversed in the high-fat/normal diet group but the $\mathrm{Ca}^{2+}$ and contractility changes where not then this suggests they are not contributing to the I/R vulnerability in the high-fat diet group.

The mice in the high-fat/normal diet group were approximately three weeks older compared to the normal and high-fat diet groups (approx. 30 vs 27 weeks). This is only a small age difference and all groups are considered as adult mice. It is not known whether these age differences between the mice in this model had an impact on vulnerability to I/R; however, ageing has been shown to augment (not attenuate) the damaging effects of I/R [19, 20]. 


\section{Conclusions}

This study shows for the first time that switching the diet from high-fat back to normal reduces vulnerability to I/R. This effect is associated with changes in catalase levels and mitochondrial morphology without altering cardiomyocyte contractility or $\mathrm{Ca}^{2+}$ transients. Therefore, the increased vulnerability to I/R after feeding mice high-fat diet is likely to be related to the elevated catalase expression and mitochondrial fragmentation.

\section{Acknowledgments}

The authors would like to thank Dr Anita Thomas for her help maintaining the mice. This work was funded by the British Heart Foundation grant number FS/11/57/28936. We also acknowledge the help of members of the National Institute for Health Research Bristol Biomedical Research Unit in Cardiovascular Disease.

\section{Disclosure Statement}

The authors declare that there are no conflicts of interest.

\section{References}

1 Littlejohns B, Pasdois P, Duggan S, Bond AR, Heesom K, Jackson CL, Angelini GD, Halestrap AP, Suleiman MS: Hearts from Mice Fed a Non-Obesogenic High-Fat Diet Exhibit Changes in Their Oxidative State, Calcium and Mitochondria in Parallel with Increased Susceptibility to Reperfusion Injury. PloS One 2014;9:e100579.

2 Liu J, Lloyd SG: High-fat, low-carbohydrate diet alters myocardial oxidative stress and impairs recovery of cardiac function after ischemia and reperfusion in obese rats. Nutr Res 2013;33:311-321.

3 Panagia M, Gibbons GF, Radda GK, Clarke K: PPAR-alpha activation required for decreased glucose uptake and increased susceptibility to injury during ischemia. Am J Physiol Heart Circ Physiol 2005;288:H26772683.

4 Thakker GD, Frangogiannis NG, Zymek PT, Sharma S, Raya JL, Barger PM, Taegtmeyer H, Entman ML, Ballantyne CM: Increased myocardial susceptibility to repetitive ischemia with high-fat diet-induced obesity. Obesity (Silver Spring) 2008;16:2593-2600.

5 Hill JO, Dorton J, Sykes MN, Digirolamo M: Reversal of dietary obesity is influenced by its duration and severity. Int J Obes 1989;13:711-722.

6 Guo J, Jou W, Gavrilova O, Hall KD: Persistent diet-induced obesity in male C57BL/6 mice resulting from temporary obesigenic diets. PLoS One 2009;4:e5370.

7 Wang HT, Liu CF, Tsai TH, Chen YL, Chang HW, Tsai CY, Leu S, Zhen YY, Chai HT, Chung SY, Chua S, Yen CH, Yip HK: Effect of obesity reduction on preservation of heart function and attenuation of left ventricular remodeling, oxidative stress and inflammation in obese mice. J Transl Med 2012;10:145.

8 Briggs DI, Lockie SH, Wu Q, Lemus MB, Stark R, Andrews ZB: Calorie-restricted weight loss reverses highfat diet-induced ghrelin resistance, which contributes to rebound weight gain in a ghrelin-dependent manner. Endocrinology 2013;154:709-717.

-9 Radonjic M, Wielinga PY, Wopereis S, Kelder T, Goelela VS, Verschuren L, Toet K, van Duyvenvoorde W, van der Werff van der Vat B, Stroeve JH, Cnubben N, Kooistra T, van Ommen B, Kleemann R: Differential effects of drug interventions and dietary lifestyle in developing type 2 diabetes and complications: a systems biology analysis in LDLr-/- mice. PloS One 2013;8:e56122.

10 Khaliulin I, Halestrap AP, Suleiman MS: Temperature preconditioning is optimal at 26 degrees C and confers additional protection to hypothermic cardioplegic ischemic arrest. Exp Biol Med (Maywood) 2011;236:736-745. 
11 Williams H, Kerr PM, Suleiman M, Griffiths EJ: Differences in the calcium-handling response of isolated rat and guinea-pig cardiomyocytes to metabolic inhibition: implications for cell damage. Exp Physiol 2000;85:505-510.

12 Halestrap AP, Pasdois P: The role of the mitochondrial permeability transition pore in heart disease. Biochim Biophys Acta 2009;1787:1402-1415.

13 Rindler PM, Plafker SM, Szweda LI, Kinter M: High dietary fat selectively increases catalase expression within cardiac mitochondria. J Biol Chem 2013;288:1979-1990.

-14 Ong SB, Hausenloy DJ: Mitochondrial morphology and cardiovascular disease. Cardiovasc Res 2010;88:1629.

15 Ong SB, Subrayan S, Lim SY, Yellon DM, Davidson SM, Hausenloy DJ: Inhibiting mitochondrial fission protects the heart against ischemia/reperfusion injury. Circulation 2010;121:2012-2022.

16 Howarth FC, Qureshi MA, Gbewonyo AJ, Tariq S, Adeghate E: The progressive effects of a fat enriched diet on ventricular myocyte contraction and intracellular Ca2+ in the C57BL/6J mouse. Mol Cell Biochem 2005;273:87-95.

17 Cheng Y, Li W, McElfresh TA, Chen X, Berthiaume JM, Castel L, Yu X, Van Wagoner DR, Chandler MP: Changes in myofilament proteins, but not $\mathrm{Ca} 2+$ regulation, are associated with a high-fat diet-induced improvement in contractile function in heart failure. Am J Physiol Heart Circ Physiol 2011;301:H14381446.

18 Bassani JW, Bassani RA, Bers DM: Relaxation in rabbit and rat cardiac cells: species-dependent differences in cellular mechanisms. J Physiol 1994;476:279-293.

19 Azhar G, Gao W, Liu L, Wei JY: Ischemia-reperfusion in the adult mouse heart influence of age. Exp Gerontol 1999;34:699-714.

20 Lesnefsky EJ, Gallo DS, Ye J, Whittingham TS, Lust WD: Aging increases ischemia-reperfusion injury in the isolated, buffer-perfused heart. J Lab Clin Med 1994;124:843-851. 